\title{
Evaluation of prognostic factors following flow-cytometric DNA analysis after cytokeratin labelling: I. Breast cancer
}

\author{
Pauline Wimberger ${ }^{\mathrm{a}, *}$, Peter Hillemanns ${ }^{\mathrm{b}}$, Thomas Kapsner ${ }^{\mathrm{c}}$, Hermann Hepp ${ }^{\mathrm{b}}$ and Rainer Kimmig ${ }^{\mathrm{a}}$ \\ ${ }^{a}$ Department of Obstetrics and Gynecology, University of Essen, Hufelandstr. 55, D-45122 Essen, Germany \\ ${ }^{\mathrm{b}}$ Department of Obstetrics and Gynecology, Ludwig-Maximilians-University, Marchioninistr. 15, \\ D-81377 Munich, Germany \\ ${ }^{\mathrm{c}}$ Department of Medical Informatics, Biometry and Epidemiology (IBE), Ludwig-Maximilians-University, \\ Marchioninistr. 15, D-81377 Munich, Germany
}

Received March 2002

Accepted 24 July 2002

\begin{abstract}
In gynecologic oncology valid prognostic factors are necessary to estimate the course of disease and to define biologically similar subgroups for analysis of therapeutic efficacy. The presented study is a prospective study concerning prognostic significance of DNA ploidy and S-phase fraction in breast cancer following enrichment of tumor cells by cytokeratin labelling. Epithelial cells were labeled by FITC-conjugated cytokeratin antibody (CK 5, 6, 8, and CK 17) prior to flow cytometric cell cycle analysis in 327 fresh specimens of primary breast cancer.

Univariate analysis in breast cancer detected the prognostic significance of DNA-ploidy, S-phase fraction and CV (coefficient of variation) of $\mathrm{G}_{0} \mathrm{G}_{1}$-peak of tumor cells for clinical outcome, especially for nodal-negative patients. Multivariate analysis could not confirm prognostic evidence of DNA-ploidy and S-phase fraction.

In conclusion, in breast cancer no clinical significance for determination of DNA-parameters was found.
\end{abstract}

\section{Introduction}

Prognostic factors are an important basis for optimal choice of therapeutic strategy. Prognostic factors are important to decide whether additional adjuvant therapeutic modalities are necessary. They render an individual care for patients with gynecologic malignancies. In addition to known classic prognostic factors like clinical stage, operability and histopathologic results many so called "prognostic factors" of the morphologic and molecular genetic domain were investigated in the last decades. New studies showed that grading and classic histopathological parameters are no reliable predictors of outcome in individual patients for example in oral leukoplakia and oral carcinoma [1].

\footnotetext{
* Corresponding author: Pauline Wimberger, M.D., Department of Gynecology and Obstetrics, University of Essen, Hufelandstr. 55, 45122 Essen, Germany. Tel.: +49 201723 0; Fax: +49 201723 5689; E-mail: pauline.wimberger@med.uni-essen.de.
}

Chromosomal rearrangements represent an early step in the initiation of tumorigenesis. The regulation of growth's control mechanisms is changed following alteration of the expression of certain genes. Alteration of genetic factors is often accompanied by quantitative changes of DNA content such as the p53 gene alteration, which is associated with DNA aneuploidy [2].

All studies investigating DNA content by flow cytometry were performed without identification of tumor cells prior to DNA analysis. Several studies indicate that mutations in genes controlling chromosome segregation during mitosis and centrosome abnormalities play a critical role in causing chromosome instability in cancer [3]. And chromosomal aberrations seem to occur exclusively in aneuploid tumor cell lines [3]. It has been shown for different carcinomas that cytokeratin staining is able to detect epithelial tumor cells $[4,5]$. For breast carcinoma $[4,6]$ and cervical carcinoma [7], about $20 \%$ of DNA-aneuploid tumor sub- 
populations are missed without enrichment for cytokeratin positive cells. In addition, S-phase fraction can be determined more accurately [6-10]. Multiparameter analysis has been recommended for future flow cytometric DNA analysis [11,12]. The presented prospective study investigated the prognostic significance of DNA ploidy and S-phase fraction in breast cancer following enrichment of tumor cells by cytokeratin labelling.

\section{Materials and methods}

Fresh tumor tissue of carcinomas of the breast ( $n=327$ ) were dissociated by combined mechanical/enzymatic method [13] as described previously in detail [14]. In short, disaggregation of tissue was performed in a prewarmed enzyme mixture in Dulbecco's phosphate buffered saline (PBS) consisting of $2 \mathrm{mg} / \mathrm{ml}$ trypsin (type III, 10,000 U/mg protein, Sigma chemicals), $2 \mathrm{mg} / \mathrm{ml}$ collagenase (type I-S $180 \mathrm{U} / \mathrm{mg}$ solid, Sigma chemicals) and $0.2 \mathrm{mg} / \mathrm{ml}$ DNase (type I, $1548 \mathrm{U} / \mathrm{mg}$ protein, Sigma chemicals) at $37^{\circ} \mathrm{C}$ for 10 minutes. Cell number and viability of the single cell suspension was determined by cell counter and trypan blue exclusion prior to fixation in $70 \%$ methanol $\left(-20^{\circ} \mathrm{C}\right)$. Following resuspension of $5 \times 10^{4}$ to $2 \times 10^{5}$ cells in $0.5 \mathrm{ml} \mathrm{PBS} / 5 \%$ fetal calf serum (FCS) cells were labeled for cytokeratins 5, 6, 8 and 17 using FITC conjugated monoclonal mouse anti-human cytokeratin antibodies (Dako CK1/DAKO-CK (Dakopatts A/S); $100 \mu \mathrm{g} \mathrm{IgG1/ml}$, dilution $1: 20$, at room temperature, 30 min, dark). Following centrifugation and washing steps cells were stained for DNA with $0.5 \mathrm{ml}$ PBS containing propidium iodide $(50 \mu \mathrm{g} / \mathrm{ml})$ and RNase (Sigma, type I-AS, $1 \mathrm{mg} / \mathrm{ml}$ ) at $37^{\circ} \mathrm{C}$ for 10 minutes. Negative controls were performed identically using a FITC conjugated mouse IgG1 isoantibody. Female human lymphocytes and HeLa cells processed in the same way served as controls for ploidy, cytokeratin staining and coefficient of variation. 10,000 to 40,000 cells per sample were measured in a FACScan flowcytometer $488 \mathrm{~nm}$ argon laser, Beckton Dickinson (BD) equipped with Hewlett Packard hardware and a pulse processor for doublet discrimination. Data acquisition and analysis was performed with the Cellfit software of BD. DNA ploidy was determined according to Hiddemann et al. [15]. Tumor proliferation was estimated by calculating percentages of $\mathrm{G}_{0} \mathrm{G}_{1^{-}}, \mathrm{S}-$, and $\mathrm{G}_{2} \mathrm{M}$-phase of each individual analyzable stemline using the SOBR method (sum of broadened rectangles). For details of quality control compare Kimmig et al. 1994 [6].

\subsection{Patients}

\subsubsection{Breast cancer}

The clinical course of breast cancer was analyzed in $240 / 327$ patients with primary breast cancer, thereby 319 were of ductal and 8 of lobular type. For prospective analysis of recurrence free survival (RFS) and cancer specific survival (CSS) we excluded breast cancer patients with only local excision $(n=1)$, preoperative chemotherapy $(n=2)$, primary metastases $(n=39)$, contralateral breast cancer $(n=15)$, patients with secondary malignancies, independent on time interval to breast cancer diagnosis $(n=18)$, ductal carcinoma in situ $(n=4)$ and invasive carcinoma lobulare $(n=8)$.

Table 1

Distribution of clinical histopathological prognostic factors in patients with primary breast cancer $(n=327)$. The distribution of patients with prospective analysis are documented in brackets

\begin{tabular}{|c|c|c|c|c|c|}
\hline Tumor size & pTis & pT1 & $\mathrm{pT} 2$ & pT3 & pT4 \\
\hline number $(n)$ & $4\{0\}$ & $79\{60\}$ & $162\{129\}$ & $23\{18\}$ & $59\{33\}$ \\
\hline Lymph node involvement & pNO & \multicolumn{2}{|c|}{$\mathrm{pN} 1(1-3 \mathrm{Ln})$} & $\mathrm{pN} 1(>3 \mathrm{Ln})$ & nd \\
\hline number $(n)$ & $138\{108\}$ & \multicolumn{2}{|c|}{$78\{63\}$} & $97\{70\}$ & $14\{0\}$ \\
\hline Grading & 1 & \multicolumn{2}{|c|}{2} & 3 & 4 \\
\hline number $(n)$ & $8\{7\}$ & \multicolumn{2}{|c|}{$175\{131\}$} & $142\{100\}$ & $2\{2\}$ \\
\hline Estrogen receptors & negative & \multicolumn{3}{|c|}{ positive } & \\
\hline number $(n)$ & $128\{100\}$ & \multicolumn{3}{|c|}{$199\{140\}$} & \\
\hline Progestagen receptors & negative & \multicolumn{3}{|c|}{ positive } & \\
\hline number $(n)$ & $134\{100\}$ & \multicolumn{3}{|c|}{$193\{140\}$} & \\
\hline Metastasis & M0 & \multicolumn{3}{|c|}{ M1 } & \\
\hline number $(n)$ & $288\{240\}$ & \multicolumn{3}{|c|}{$39\{0\}$} & \\
\hline
\end{tabular}


Table 2

Distribution of therapy modalities in patients with primary breast cancer. Only patients analyzed prospectively were determined $(n=240)$.

\begin{tabular}{lc}
\hline Therapy of breast cancer & Number $(n)$ \\
\hline Breast conserving therapy and radiation & 73 \\
Mastectomy and lymphonodectomy & 166 \\
Ablatio simplex & 1 \\
Adjuvant radiation & $21 / 166$ \\
Adjuvant chemotherapy & 92 \\
Adjuvant hormone therapy & 64 \\
\hline
\end{tabular}

Prospective analysis was, therefore, performed in 240 patients with median age of 57 years (range 33.4 to 86.9 years).

The distribution of five clinico-histopathological parameters for the 327 patients is shown in Table 1; thereby patients were analyzed by univariate and multivariate analysis (noticed in brackets). Therapy modalities for the last mentioned group was demonstrated in Table 2. Cut off for positive or negative steroid receptors were $10 \mathrm{fmol} / \mathrm{mg}$ protein.

The median follow up was 1413 days (25th percentile 778 days / 75th percentile 2114 days). 240 patients had a complete follow up and 76 (32\%) of these patients showed locoregionary relapse or metastasis, whereas intramammarian relapse after breast preserving therapy was not valued. 57 patients died, 48 (20\%) as result of their disease status.

\subsubsection{Statistics}

The experimental data were analyzed with using SAS (SAS Institute Inc., Cary NC) on a UNIX work station. The different proportions of DNA-aneuploid tumors found with gating for cytokeratin positive cells compared to those without gating were described using a two-by-two table. As no definitive reference test for determination of ploidy was available, the results obtained with gating could only be described in relation to our results without gating. The McNemar test was used to test the null hypothesis that the results of the two methods are distinguishable. In order to compare proportions from cell counts obtained by cell cycle analysis with the statistical test they were transformed to the angular scale using an arcsin transformation. The transformed proportions were compared with the use of a paired $t$-test [16]. Calculation of recurrence free survival (RFS) and tumor dependent overall survival (CSS) was estimated according to the Kaplan-Meier method. The independent prognostic significance of each factor was evaluated by Cox regression model. Two-tailed $p$-values are given. $P$-values less than 0.05 were considered statistically significant.

\section{Results}

\subsection{Breast cancer}

In 327 patients with breast cancer, single cell suspensions of tumors were investigated.

The analysis of all cell suspensions showed that 202 of the tumor specimens (62\%) were DNA-aneuploid without cytokeratin labelling of tumor cells, whereas $250(76.5 \%)$ were detected as DNA-aneuploid following cytokeratin staining. The number of tumor specimens with more than one DNA-subpopulation increased from 46 ( $14 \%$ of $n=327)$ to $74(23 \%)$.

The distribution of the DNA-indices of cytokeratinlabeled tumor cells is presented in detail elsewhere [14].

\subsubsection{Correlation of DNA-ploidy, DNA-index, CV and cell cycle fraction with classic clinical- histopathological prognostic factors in breast cancer}

Patients with DNA-aneuploid tumors show more often worse differentiated tumors (G III, $p<0.0001$ ) and axillary lymph node involvement $(p=0.01)$. The same results were found for DNA-index. Tumors with a higher $\mathrm{CV}$ were significantly more often estrogen- or progestagen receptor negative $(p=0.05, p=0.003)$. The S-phase fraction correlated significantly with tumor size $(p=0.02)$, lymph node status $(p=0.02)$, estrogen- $(p=0.006)$, progestagen receptor $(p<$ $0.0001)$ and grading $(p<0.0001)$. No correlation for $\mathrm{G}_{2} \mathrm{M}$-phase fraction to clinical-histopathologic parameters was assessed.

\subsection{Prognostic significance of DNA-ploidy and $S$-phase fraction in breast cancer in comparison to classic clinical-histopathologic prognostic factors}

\subsubsection{Prognostic significance of classic clinical- histopathologic prognostic factors}

Recurrence-free survival (RFS) and cancer specific overall survival (CSS) were performed in 240 patients with primary breast carcinoma. The axillary lymph node involvement and the histopathologic grading showed the highest discrimination capacity and statistic significance for RFS and CSS. Nearly all patients with metastatic disease of breast cancer will die, so the results for RFS are predictors for CSS because of only limited follow-up. After a median follow-up time of 46.5 months RFS of patients without axillary lymph 
node involvement was $79.6 \%$ and in case of grading of G1 or G2 $76.8 \%$, but in case of lymph node involvement RFS was only $59.1 \%$, respectively $58.0 \%$ for G3 ( $p=0.0003 ; p=0.0002)$. The corresponding data for CSS were $90.7 \%$ and $88.4 \%$ versus $71.2 \%$ and $69 \%$ $(p=0.0001)$. But also age, tumor size, estrogen- and progestagen receptors were univariate significant prognostic factors.

For estimation of time course and relation of censored patients to patients with relapse or tumor dependent death Kaplan-Meier curves were shown for the parameters tumor size, lymph node status, grading and progestagen receptor for RFS and CSS.

\subsubsection{Prognostic significance of classic clinical- histopathologic prognostic factors in dependence on lymph node status}

The high prognostic significance of axillary lymph node status is known. But unfortunately, also lymph node negative patients show relapse in $30 \%$. These patients did not get obligatory any adjuvant treatment in the past, so it is very important to identify further prognostic factors for these patients. Thereby patients with and without lymph node involvement were analyzed separately.

In case of negative nodes $(n=108)$ all mentioned factors, especially histopathologic grading and hormone receptors, were of prognostic significance in univariate analysis apart from age.

Also for nodal negative patients Kaplan-Meier curves were shown for estimation of time course and relation of censored patients to patients with relapse or tumor dependent death for the parameters tumor size, grading and progestagen receptor for RFS and CSS. Whereas tumor size ( $p=0.004)$ seems to be the predominant parameter concerning RFS in addition to histopathologic grading ( $p=0.002$ ), the predominant parameter in CSS was the progestagen receptor $(p=0.008)$.

In contrast, for lymph node positive patients ( $n=$ 132) concerning RFS no significant prognostic factor $(p>0.05)$ was detected, but the significance for most of the prognostic factors concerning CSS was maintained. The histopathologic grading was the most important factor, too $(p=0.02)$. But thereby in contrast to the nodal negative patients, also age showed a borderline significant prognostic significance.

\subsection{Prognostic significance of DNA-ploidy, DNA-index and $C V$}

The prognostic significance of DNA-ploidy for RFS and CSS was investigated with respect to three parameters:
1. DNA-ploidy differentiated as DNA-diploid with inclusion of near peridiploid tumors (DNA-content $>1.8 \mathrm{c}$ and $<2.2 \mathrm{c}$ ) and DNA-aneuploid (each other DNA-content). With cytokeratin labelling of tumor cells a higher detection rate of DNA-aneuploid subpopulation was present. For estimation of clinical relevance the prognostic significance of DNA-ploidy was analyzed with and without cytokeratin labelling.

2. DNA-index (relation of DNA-content of the tumor cell to DNA-content of normal DNA-diploid somatic cells of the same organism): Tumors with a DNA-index $\leqslant 1.3$ were compared to tumors with a DNA-index $>1.3$.

3. The CV as index for "microvariance" of DNAcontent was stratified at median.

Univariate analysis found a prognostic relevance for DNA-ploidy for CSS with $91.2 \%$ versus $76.5 \%$ for DNA-diploid and DNA-aneuploid tumors $(p=0.038)$. The difference of $77.2 \%$ to $65.6 \%$ in RFS was not statistically significant. Without cytokeratin labelling similar results were detected. DNA-index discriminated worse and reached no significance, in contrast to the $\mathrm{CV}$, that showed the highest significance for RFS (76.9\% versus $59.8 \% ; p=0.019$ ) and also for CSS (87.6\% versus $72.6 \% ; p=0.018)$.

For estimation of time course and relation of censored patients to patients with relapse or tumor dependent death Kaplan-Meier curves were shown for the parameters DNA-ploidy with and without cytokeratin labelling, CV and S-phase fraction for RFS (Fig. 1) and CSS (data not shown).

Although after an observation time of 30 months a trend of higher RFS for patients with DNA-diploid tumors was detected, this trend had no statistic significance, but after cytokeratin labelling differentiation seemed to be somewhat better. Whereas a significant better RFS for patients with lower CV was observed $(p=0.0019)$, no difference was found for patients with respect to high or low proliferation of tumor. Concerning CSS similar results were detected for CV and S-phase fraction, whereas DNA-ploidy is a significant prognostic factor $(p=0.03)$. And the discrimination after cytokeratin labelling at similar significance is also somewhat better.

\subsection{Prognostic significance of DNA-ploidy, $D N A$-index and $C V$ in dependence on lymph node status}

In nodal negative patients recurrence-free survival after cytokeratin labelling in DNA-aneuploid tumors 

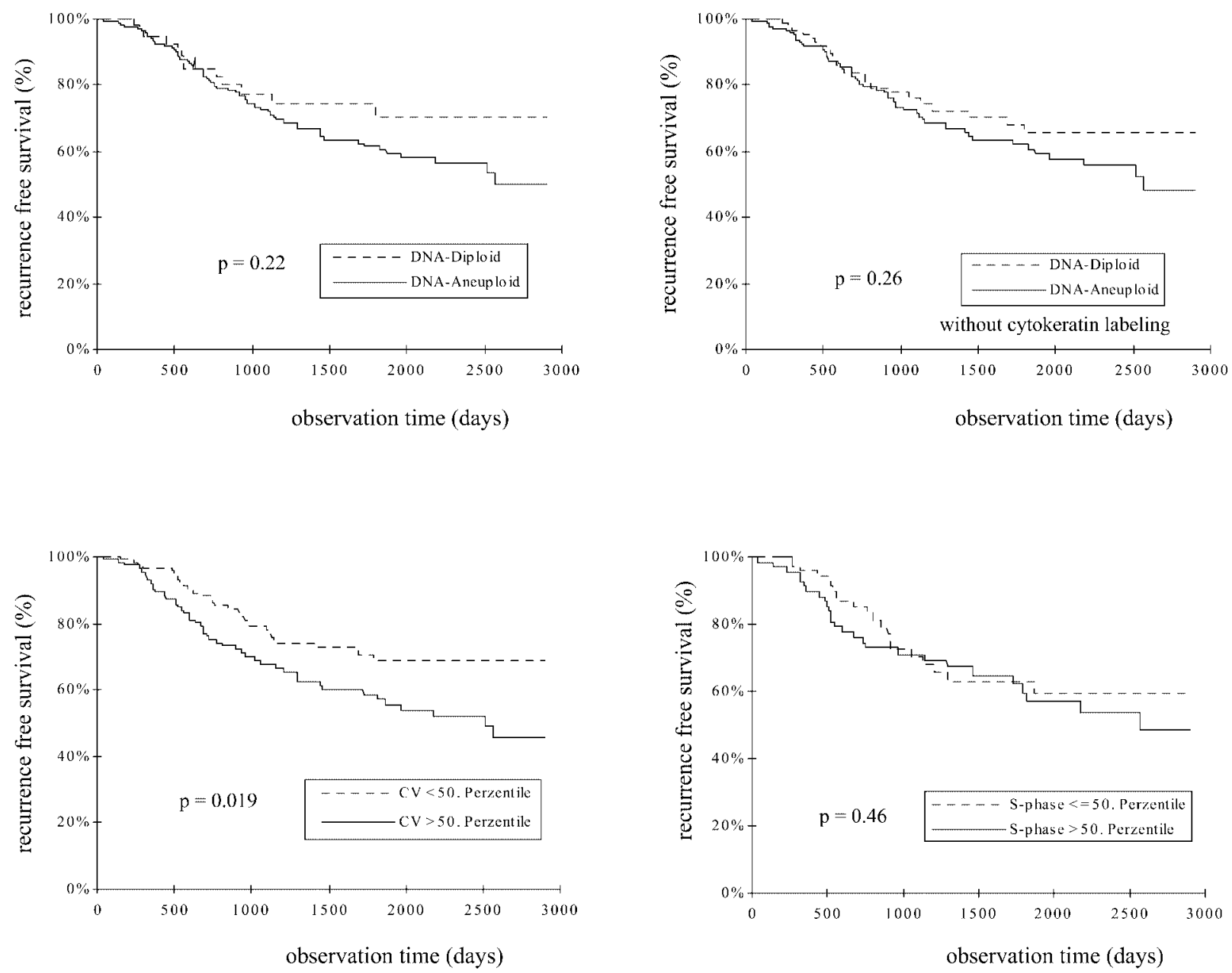

Fig. 1. Primary breast cancer $(n=240)$. Kaplan-Meier curves for RFS in dependence on DNA-ploidy (with and without cytokeratin labelling), $\mathrm{CV}$ and S-phase fraction were performed. The significance level $(p)$ was determined by univariate analysis with the log-rank test.

decreased to $74.3 \%$ from $91.2 \%$ in DNA-diploid tumors $(p=0.052)$. Without cytokeratin labelling the difference is lower $(86.8 \%$ versus $72.3 \%)$ and is not significant $(p=0.096)$. DNA-index has no prognostic significance.

For presentation of time course and relation of censored patients to patients with relapse or tumor dependent death Kaplan-Meier curves were shown and a better RFS for patients with DNA-diploid tumors and low $\mathrm{CV}$ of $\mathrm{G}_{0} \mathrm{G}_{1}$-peak was demonstrated (Fig. 2) and the same was found for CSS (data not shown). But for CSS DNA-ploidy has no statistic significance. Tumors with low S-phase fraction tend to have a better RFS and CSS, but the differences were not significant.

In contrast to lymph node negative patients, lymph node positive patients show no prognostic significance concerning the mentioned parameters. Whereas
DNA-ploidy and DNA-index show no differences in subgroups concerning RFS, worse data for DNAaneuploid tumors for CSS were found, but without any statistic significance. The greatest difference was present for $\mathrm{CV}$, but again without any significance in log-rank test $(p=0.08)$.

\subsection{Prognostic significance of $S$-phase fraction, $G_{2} M$-phase fraction with and without identification of tumor cells by cytokeratin labelling}

The prognostic significance of S-phase fraction, $\mathrm{G}_{2} \mathrm{M}$-phase fraction were evaluated separately for results with and without cytokeratin labelling.

For stratification in low and high proliferated tumors, the known parameters were divided into quar- 

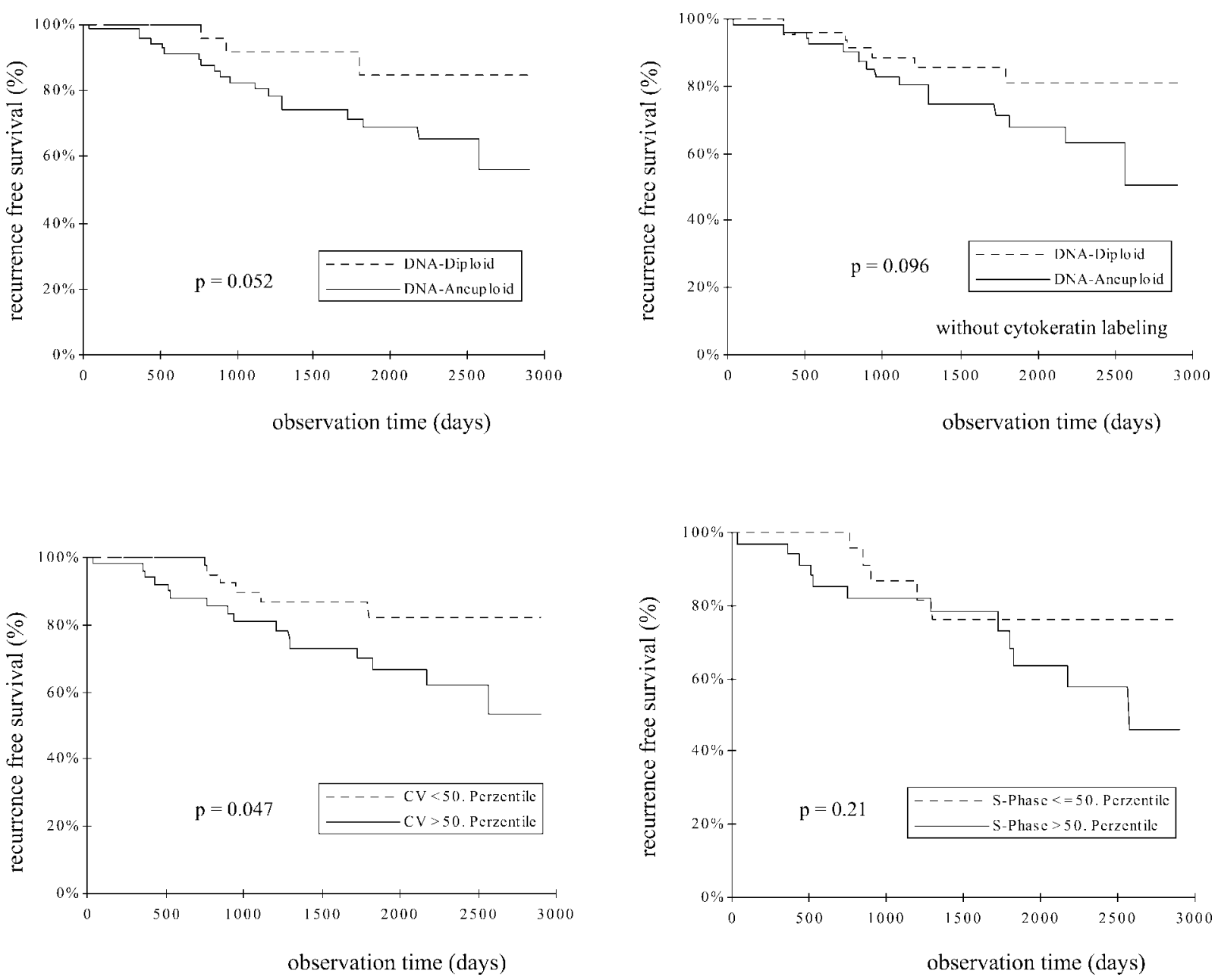

Fig. 2. Node-negative breast cancer $(n=108)$. Kaplan-Meier curves for RFS in dependence on DNA-ploidy (with and without cytokeratin labelling), CV and S-phase fraction were performed. The significance level $(p)$ was determined by univariate analysis with the log-rank test.

tiles. For all parameters an analysis was performed with division at median and the inferior 3 quartiles versus the highest quartile; in addition, the lowest quartile versus the 3 upper quartiles were analyzed for the S-phase fraction of cytokeratin positive cells.

A trend of lower RFS and CSS was detected for higher proliferating tumors. The trend was only of borderline significance for RFS in S-phase fraction with a cut-off at the lowest quartile versus the rest (81.6\% versus $61.7 \% ; p=0.058)$, and median of $\mathrm{G}_{2} \mathrm{M}$-phase fraction ( $76 \%$ versus $57 \% ; p=0.056$ ). Whereas $81.6 \%$ of the patients with a S-phase fraction in the lowest quartile survive recurrence-free, only $52.9 \%$ were found with S-phase fraction in the uppermost quartile. This was only present for cell cycle parameters of cytokeratin-positive cells. The analysis of all cells detected no significance.

\subsection{Prognostic significance of S-phase fraction, $G_{2} M$-phase fraction with and without identification of tumor cells by cytokeratin labelling in dependence on lymph node status}

Nodal negative patients showed a decrease of RFS from $88.2 \%$ to $50.0 \%$ and CSS from $100 \%$ to $75 \%$ from the lowest to highest quartile of S-phase fraction in dependence on lymph node status. However, only the proliferation fraction $\mathrm{S}+\mathrm{G}_{2} \mathrm{M}$ reached statistic significance after cytokeratin labelling for the highest quartile versus the rest with an RFS of only $47.1 \%$ for high proliferating tumors versus $84.3 \%$ for medium and low proliferating tumors $(p=0.03)$. No statistic significance was found for all parameters in case of no cytokeratin labelling. 
Lymph node positive patients show a trend for better prognosis for RFS and CSS in lower proliferating tumors. RFS decreased from $70 \%$ in case of low $\mathrm{S}+\mathrm{G}_{2} \mathrm{M}$-phase fraction to $48.8 \%$ in case of higher $\mathrm{S}+\mathrm{G}_{2} \mathrm{M}$-phase fraction. A similar result was detected for $\mathrm{G}_{2} \mathrm{M}$-phase fraction $(71.8 \%$ versus $47.4 \%)$, whereas in S-phase fraction a difference was observed only for the first quartile versus the rest $(70 \%$ versus $56.1 \%$ ). This was not statistically significant. An analogue situation was present for CSS.

\subsection{Prognostic significance of DNA-ploidy and $S$-phase fraction in breast cancer in comparison to classic prognostic factors: multivariate analysis}

\subsubsection{All patients}

Because of close relationship between prognostic factors, a multivariate analysis was necessary for evaluation of independent prognostic significance.

We therefore performed COX-multivariate analysis for all statistically significant parameters in univariate analysis. Analyzed were age of patients, tumor size (pT), lymph node status (pN), grading, estrogen- and progestagen receptor, DNA-ploidy and $\mathrm{CV}$ of $\mathrm{G}_{0} \mathrm{G}_{1}$ peak.

Variables of 236 patients with primary breast cancer were presented in Table 3.

For RFS tumor size, axillary lymph node status, progestagen receptor and $\mathrm{CV}$ of $\mathrm{G}_{0} \mathrm{G}_{1}$ peak of tumor cells were significant with a relative risk (RR) between $1.6(\mathrm{CV})$ and 2.6 (progestagen receptor). For patients younger than 52 years, CSS was significantly worse with a relative risk of 2.1 and the RR in case of lymph node positivity is even 2.4 . The grading is identified also as an independent prognostic factor with an RR of 1.9. The highest relative risk showed progestagen receptor, with an RR of 2.7 .

\subsubsection{Patients with nodal negative or nodal positive breast cancer}

In nodal negative patients, tumor size, grading, progestagen receptors and $\mathrm{CV}$ of $\mathrm{G}_{0} \mathrm{G}_{1}$ peak were of independent significance for RFS with a RR between 3.8 (progestagen receptor) and 4.0 (tumor size). For CSS only the grading showed a significant correlation after multivariate analysis with an RR of 6.5 $(p=0.007)$.

Multivariate analysis in nodal positive patients detected no significant prognostic factor for RFS. In CSS a worse tumor differentiation showed an RR of 2.1 $(p=0.03)$.

\section{Discussion}

\subsection{Prognosis of breast cancer}

Breast cancer is the most common malignancy of women. One of nine women gets this tumor [17]. With respect to health and business it is very important which therapy will be performed and when. Therefore, a correct estimation of tumor biology and course of disease is necessary. Half of the women with primary operable breast cancer could be cured by operation only. These patients would not have any benefit by adjuvant treatment. The most important predictor of outcome for these patients is the lymph node involvement [18, 19]. In case of negative lymph nodes only $30 \%$ of patient show relapse within 10 years [20]. Tumor size is an important prognostic factor in node negative patients; the five year survival decreases from $>99 \%$ in case of tumor diameter of $<0.5 \mathrm{~cm}$ to $82 \%$ for tumors $>5 \mathrm{~cm}$ [21]. The recurrence-free survival within 20 years decreases in tumors of 1.7 to $2 \mathrm{~cm}$ diameter to

Table 3

Primary breast cancer $(n=236)$. Cox-multivariate analysis of univariate prognostic relevant parameters for recurrence-free survival (RFS) and tumor-dependent overall survival (CSS). For all parameters multivariate analysis were performed. For all parameters the value of effect of covariable estimation $(\beta)$, standard deviation $(\sigma)$, relative risk (RR) and the belonging $95 \%$ confidence interval $(95 \% \mathrm{CI})$ was determined

\begin{tabular}{|c|c|c|c|c|c|c|c|c|c|c|}
\hline \multirow{2}{*}{$\begin{array}{l}\text { Breast cancer } \\
n=236\end{array}$} & \multicolumn{5}{|c|}{ RFS } & \multicolumn{5}{|c|}{ CSS } \\
\hline & $\beta$ & $\sigma$ & $p$ & $\mathrm{RR}$ & $95 \% \mathrm{CI}$ & $\beta$ & $\sigma$ & $p$ & $\mathrm{RR}$ & $95 \% \mathrm{CI}$ \\
\hline Age & -0.38 & 0.24 & 0.19 & & & -0.76 & 0.31 & 0.01 & 2.13 & $1.17-3.91$ \\
\hline Tumor size (pT) & 0.58 & 0.19 & 0.002 & 1.79 & $1.23-2.58$ & 0.58 & 0.24 & 0.02 & 1.79 & $1.11-2.87$ \\
\hline Lymphnode status & 0.59 & 0.27 & 0.03 & 1.81 & $1.07-3.05$ & 0.88 & 0.38 & 0.02 & 2.42 & $1.16-5.04$ \\
\hline Grading & 0.33 & 0.27 & 0.22 & & & 0.64 & 0.34 & 0.06 & 1.90 & $0.98-3.71$ \\
\hline Estrogen receptor & 0.14 & 0.36 & 0.70 & & & 0.95 & 0.38 & 0.92 & & \\
\hline Gestagen receptor & -0.77 & 0.24 & 0.001 & 2.15 & $1.35-3.43$ & -0.99 & 0.31 & 0.001 & 2.70 & $1.48-4.93$ \\
\hline DNA-ploidy & -0.03 & 0.33 & 0.91 & & & 0.33 & 0.49 & 0.51 & & \\
\hline $\mathrm{CV} \mathrm{G}_{0} \mathrm{G}_{1}$-peak & 0.48 & 0.25 & 0.05 & 1.62 & $0.99-2.63$ & 0.61 & 0.32 & 0.06 & 1.84 & $0.98-3.46$ \\
\hline
\end{tabular}


$59 \%$ compared to $88 \%$ in tumors smaller than $1 \mathrm{~cm}$ diameter [22]. Also the histopathological grading according to Bloom and Richardson [23] correlates with risk for relapse. Risk for metastasis within 5 years increases from $2.3 \%$ (grading I) to $15.2 \%$ (grading II) [24]. The expression of steroid receptors showed an advantage of $8-10 \%$ in recurrence-free and overall survival within 5 years [17]. Up to now no parameter was found, also no "new prognostic factor" like HER2 oncogene, kathepsin D and others, that result in a definitive categorization in relapse risk versus no relapse risk $[17,25]$. Our investigation confirmed significant prognostic factors like lymph node status, tumor size, grading and progestagen receptor in multivariate analysis.

\subsection{Prognostic significance of DNA-parameters in comparison to clinical histopathological prognostic factors}

\subsubsection{DNA-ploidy}

The published data concerning prognostic significance of DNA-ploidy are numerous, but controversial. As early as 1980 it was shown that patients with DNA-diploid breast cancer survive longer than DNAaneuploid ones [26]. The first euphoria decreased as other research groups could not reproduce these results. Cause for controversial results could be the varying methods of tissue processing (paraffin versus frozen sections versus fresh specimen), varying tissue dissociation and acquisition of data. Another reason for controversial results are often small patient numbers and because of this very different patient collectives concerning tumor histology, stage, steroid receptor expression and age.

Of great relevance is also good quality of follow-up, statistics and correct documentation. In addition, it is crucial to perform not only univariate but also multivariate analysis.

Most studies could not evaluate any multivariate significant prognostic significance for DNA-ploidy [27], but others found especially for nodal negative patients a multivariate significance [28,29]. In contrast, Canizares et al. declared DNA-ploidy as independent prognostic factor for node-positive and also nodenegative patients [30]. A prognostic factor that is dependent on method and chosen collective is not useful for clinical routine. Thus it does not seem understandable that DNA-ploidy was declared as established prognostic factor for breast cancer [17] and as important factor for estimation of relapse risk for nodal negative patients [25]. Our investigation in 327 patients with breast cancer and 240 patients is one of the largest collective concerning prospectively performed DNA-analysis. It is the largest study with the use of cytokeratin antibodies for identification of tumor cells. Univariate analysis found a decrease in RFS from $77.2 \%$ to $64.6 \%$ (n.s.) and for CSS from $91.2 \%$ to $76.5 \%$ ( $p=0.03$ ) for the whole collective. Whereas for nodal positive patients no significant influence of DNA-ploidy for course was detected, for nodal negative patients the RFS decreased from $91.2 \%$ to $74.3 \%$ $(p=0.05)$ and CSS decreased from $97.1 \%$ to $87.8 \%$ (n.s.). Multivariate analysis found no prognostic significance for DNA-ploidy in both groups. The high correlation of DNA-ploidy with the histopathologic grading $(p<0.0001)$ and with the axillary lymph node state $(p=0.01)$ explains the loss of prognostic significance in multivariate analysis.

\subsubsection{Variation coefficient of $G_{0} G_{1}$-peak of tumor cells}

Human solid tumors develop different DNA-alterations, that could lead to changes in genetic informations and biologic behavior. At least two different mechanisms for development of genetic instability were identified. On the one hand, the abnormalities of the p53 gene, that lead to an alteration in DNA-content of cells, that could be diagnozed as DNA-aneuploidy by flow-cytometry. On the other hand, mechanisms were identified that cause broad microsatellite instability in near diploid cells [2]. The last mentioned mechanisms result only in small changes of DNA-content. Cytogenetic examinations showed that this part of aneuploid clones in breast cancer, that has only a small deviation of DNA-content of stem cell population, could not be diagnozed by flow cytometry [31]. These subpopulations could not be assessed in a shared manner because of the limited resolution of $\mathrm{G}_{0} \mathrm{G}_{1}$-peak. Because of different DNA-contents in comparison to stem cell line and DNA-diploid cell lines a broadening of $\mathrm{G}_{0} \mathrm{G}_{1}$-peak and thereby an increase in variation coefficient results. If the genetic alteration leads to a biologic advantage in tumor growth, the CV of tumor cells should have prognostic significance. For estimation of influence of method to $\mathrm{CV}$ parallel determinations of the CV in tumor cells $(3.8 \%)$ and also of contaminating normal cells $(3.2 \%)$ were performed. In univariate analysis concerning the prognostic significance of $\mathrm{CV}$ of tumor cells, we found a significant decrease of RFS in total collective from $76.9 \%$ to $59.8 \%(p=0.02)$ and a decrease of CSS from $87.6 \%$ to $72.6 \%(p=0.02)$. Thus, in univariate analysis the $\mathrm{CV}$ was a stronger 
prognostic factor compared to DNA-ploidy. Also in multivariate analysis it was of borderline significance with a relative risk (RR) of 1.6 for RFS $(p=0.05)$ and 1.8 for CSS $(p=0.06)$. The CV showed a correlation of expression of estrogen to progestagen receptors ( $p=0.05 \pm 0.003$ ), but no correlation for the other examined parameters. Up to now the prognostic significance of this parameter has not been described by other groups.

\subsubsection{S-phase fraction}

The proliferation rate could be measured in mitotic activity as important parameter of tumor behavior about 100 years ago [32]. The thymidin labelling index (TLI) as proliferation marker was examined and his prognostic significance could be shown, especially low TLI results in good prognosis [33]. In clinical routine the TLI could not be established because fresh tissue is necessary and the method is very time intensive. The determination of S-phase fraction, as marker of tumor cell proliferation, also in fixed specimen is methodologically easier.

In most studies $\mathrm{S}$-phase fraction was detected as significant prognostic factor. However, one study found a significance only for DNA-diploid tumors. 2 other studies included DNA-ploidy and $\mathrm{G}_{2} \mathrm{M}$-phase for reaching significance [34]. A further observation was that in 3 additional studies for determination of independent prognostic significance of S-phase fraction the histopathologic grading was not included in multivariate analysis [35]. However, grading is a very important prognostic factor especially for node negative patients [17]. Various authors found a significant correlation between grading and S-phase fraction [36]. Therefore the inclusion of the grading in multivariate analysis is absolutely necessary for valid statements. One large study ( $n=802)$ with a long time of followup ( $>8$ years) included the most important histopathologic prognostic factors like tumor size, lymph node status and grading in a multivariate analysis [37]. In this study S-phase fraction was a high significant prognostic factor also in multivariate analysis (RR 2.9; $p<0.0001)$. Since this is the only study showing independent prognostic significance of S-phase fraction; it is questionable, whether therapeutic consequences of a high S-phase fraction may be drawn [38]. Our investigation confirmed the trend of a worse recurrence-free and tumor dependent overall survival. The recurrencefree survival decreased in the whole collective from $81.6 \%$ for the lowest quartile to $52.9 \%$ for the uppermost quartile and the tumor dependent overall survival decreased from 94.7 to $69.4 \%$. This difference was of borderline significance in univariate analysis; with cut off at median we found no significance. The difference in RFS of nodal negative carcinoma was $88.2 \%$ versus $50 \%$ and $100 \%$ versus $75 \%$ for tumor dependent overall survival. But the difference does not reach significance at the 0.05 level.

\subsection{Conclusion for breast cancer}

The prognostic significance of DNA-ploidy and Sphase fraction in univariate analysis is evident and plausible in view of molecular biology. However, in multivariate analysis no independent prognostic significance could be found for both factors. Even if a prognostic significance could be demonstrated in larger trials, the expected survival advantage of the prognostic better group would be to small to justify a change of therapeutic strategy. So we have no indication for determination of DNA-ploidy and S-phase fraction for clinical routine diagnostic. The parameters should only be determined to investigate scientific aspects concerning a probable predictive significance for estimation of therapy success in case of adjuvant chemotherapeutic or hormone therapy.

\section{Summary}

An ideal prognostic factor should predict the course of disease exactly. At present the well-known "classic" prognostic factors, like tumor size, lymph node involvement and grading are the most important factors.

In addition, for breast cancer for example the hormone receptor stage is an excellent prognostic factor. The flow cytometric DNA-cell cycle analysis got importance as an additional diagnostic factor for a better estimation of prognosis in different malignant tumors.

The discrepancy of results of various studies, however, is confusing concerning risk estimation.

In a prospective study we studied the influence on tumor cell enrichment by using cytokeratin antibodies by flow cytometric data of DNA-ploidy and cell cycle distribution and as second step the prognostic significance of data in correlation to classic histopathological prognostic parameters.

In 240 breast cancers we determined all the parameters with a median observation period of nearly 4 years.

The identification of DNA-aneuploid subpopulations was improved by cytokeratin labelling. Hereby $15 \%$ of former DNA-diploid classified subpopulations 
were identified as DNA-aneuploid ones after cytokeratin gating.

The S-phase fraction increased after cytokeratin labelling for $30 \%$. A significant correlation was found for DNA-ploidy, lymph node involvement and grading, correlation between S-phase fraction and tumor size the lymph node involvement, grading and steroid hormone receptor status.

Univariately, the prognostic significance of DNAploidy, S-phase fraction and variation coefficient of $\mathrm{G}_{0} \mathrm{G}_{1}$-peak of tumor cells, especially for node negative patients was assessed.

Multivariately, neither DNA-ploidy nor S-phase fraction was of prognostic significance, although an advantage in growth of tumors with higher proliferation rate and higher mutation was evident. The high correlation with classic prognostic factors may be the reason. Our results showed that there is no indication for determination of DNA-ploidy or S-phase fraction in breast cancer in clinical routine.

In breast cancer a DNA-analysis for prognostic purposes should not be performed, because neither a prognostic significance for DNA-parameters in multivariate analysis could be assessed, nor a clinical consequence will result. However, cytokeratin labelling for enrichment of tumor cells led to methodological convincing advantages for detection of DNA-aneuploid subpopulations and for detection of cell cycle fractions. Concerning prognostic statement of the determined parameters marginal advantages were detected in comparison to the analysis without cytokeratin labelling. The higher costs of cytokeratin labelling by flow-cytometric DNA-analysis therefore are not justified for clinical routine.

\section{Acknowledgements}

We wish to thank Dieta Egner and Marianne Fileki for excellent technical assistance. In addition, we thank Hans Arnholdt, M.D., and Joachim Diebold, M.D., Department of Pathology, Ludwig-MaximiliansUniversity of Munich, for analysis of the histology of the tumor specimens and analysis of cytokeratin expression of the different cellular components in tissue sections by immunohistology. The study was supported by a grant from the "Münchner Medizinische Wochenschrift" (MMW).

\section{References}

[1] J. Sudbo, W. Kildal, B. Risberg H.S. Koppang, H.E. Danielsen and A. Reith, DNA content as a prognostic marker in patients with oral leukoplakia, N. Engl. J. Med. 344 (2001), 1270-1278.

[2] S.E. Shackney and T.V. Shankey, Common patterns of genetic evolution in human solid tumors, Cytometry 29 (1997), 1-27.

[3] J. Sudbo, T. Ried, M. Bryne, W. Kldal, H. Danielsen and A. Reith, Abnormal DNAA content predicts the occurrence of carcinomas in non-dysplastic oral white patches, Oral Oncol. 37 (2001), 558-565.

[4] J.C. Van der Linden, C.J. Herman, J.G. Boenders, M.M. van de Sandt and J. Lindeman, Flow cytometric DNA content of fresh tumor specimens using keratin-antibody as second stain for two-parameter analysis, Cytometry 13 (1992), 163-168.

[5] R.J. Zarbo, D.W. Visscher and J.D. Crissman, Two-color multiparametric method for flow cytometric DNA analysis of carcinomas using staining for cytokeratin and leukocyte-common antigen, Anal. Quant. Cytol. Histol. 11 (1989), 391-402.

[6] R. Kimmig, H. Spelsberg, T. Kapsner, M. Untch and H. Hepp, Flow cytometric DNA analysis of breast cancer by two colour method using cytokeratin labelling for identification of tumour cells, Analyt. Cell. Pathol. 7 (1994), 205-215.

[7] R. Kimmig, T. Kapsner, H. Spelsberg, M. Untch and H. Hepp, DNA cell-cycle analysis of cervical cancer by flow cytometry using simultaneous cytokeratin labelling for identification of tumour cells, J. Cancer Res. Clin. Oncol. 121 (1995), 107-114.

[8] S. Wingren, O. Stal, J. Carstensen, X.F. Sun and B. Nordensjold, S-phase determination of immunoselected cytokeratincontaining breast cancer cells improves the prediction of recurrence, Breast Cancer Res. Treat. 29 (1994), 179-187.

[9] S. Wingren, O. Stal and B. Nordensjold, Flow cytometric analysis of S-phase fraction in breast carcinomas using gating on cells containing cytokeratin. South East Sweden Breast Cancer Group, Br. J. Cancer 69 (1994), 546-549.

[10] S. Wingren, O. Stal, S. Sullivan, A. Brisfors and B. Nordenskjold, S-phase fraction after gating on epithelial cells predicts recurrence in node-negative breast cancer, Int. J. Cancer 59 (1994), 7-10.

[11] L.G. Dressler and S.A. Bartow, DNA flow cytometry in solid tumors: practical aspects and clinical applications, Semin. Diagn. Pathol. 6 (1989), 55-82.

[12] D.W. Hedley, G.M. Clark, C.J. Cornelisse, D. Killander, T. Kute and D. Merkel, Consensus review of the clinical utility of DNA cytometry in carcinoma of the breast. Report of the DNA Cytometry Consensus Conference, Cytometry 14 (1993), $482-485$.

[13] E. Kedar, B. Ikejiri, G.D. Bonnard and R.B. Herberman, A rapid technique for isolation of viable tumor cells from solid tumors: use of the tumor cells for induction and measurement of cell-mediated cytotoxic responses, Eur. J. Cancer Clin. Oncol. 18 (1982), 991-1000.

[14] R. Kimmig, P. Wimberger, T. Kapsner and P. Hillemanns, Flow cytometric DNA analysis using cytokeratin labelling for identification of tumor cells in carcinomas of the breast and the female genital tract, Analyt. Cell. Pathol. 22 (2001), 165-178. 
[15] W. Hiddemann, J. Schumann, M. Andreed, B. Barlogie, C.J. Herman, R.C. Leif, B.H. Myall, R.F. Murphy and A.A. Sandberg, Convention on nomenclature for DNAA cytometry. Committee on Nomenclature, Society for Analytical Cytology, Cancer Genet. Cytogenet. 13 (1984), 181-183.

[16] G.W. Snedecor and W. Cochran, Statistical Methods, 6th edn, The Iowa State University Press, Ames, Iowa, USA, 1973, pp. 327-329.

[17] R.M. Elledge, W.L. McGuire and C.K. Oborne, Prognostic factors in breast cancer, Semin. Oncol. 19 (1992), 244-253.

[18] B. Fisher, M. Bauer, R. Margolese, R. Poisson, Y. Pilch, C. Redmond, E. Fisher, N. Wolmark, M. Deutsch and E. Montague, Five-year results of a randomized clinical trial comparing total mastectomy and segmental mastectomy with or without radiation in the treatment of breast cancer, $N$. Engl. J. Med. 312 (1985), 665-673.

[19] B. Fisher, C. Redmond, E.R. Fisher, M. Bauer, N. Wolmark, D.L. Wickerham, M. Deutsch, E. Montague, R. Margolese and R. Foster, Ten-year results of a randomized clinical trial comparing radical mastectomy and total mastectomy with or without radition, N. Engl. J. Med. 312 (1985), 674-681.

[20] P. Valagussa, G. Bonadonna and U. Veronesi, Patterns of relapse and survival following radical mastectomy, Cancer 41 (1978), 1170-1178.

[21] C.L. Carter, C. Allen and D.E. Henson, Relation of tumor size, lymph node status, and survival in 24,740 breast cancer cases, Cancer 63 (1989), 181-187.

[22] P.P. Rosen, S. Groshen, P.E. Saigo, D.W. Kinne and S. Hellman, Pathological prognostic factors in stage I (T1NOM0) and stage II (T1N1M0) breast carcinoma: a study of 644 patients with median follow-up of 18 years, J. Clin. Oncol. 7 (1989), 12391251.

[23] H.J. Bloom and W.W. Richardson, Histologic grading and prognosis in breast cancer, Br. J. Cancer 11 (1957), 359-377.

[24] V. Le Doussal, M. Tubiana Hulin, S. Friedman, K. Hacene, F. Spyratos and M. Brunet, Prognostic value of histologic grade nuclear components of Scarff-Bloom-Richardson (SBR). An improved score modification based on a multivariate analysis of 1262 invasive ductal breast carcinomas, Cancer 64 (1989), 1914-1921.

[25] W.L. McGuire, A.K. Tandon, D.C. Allred, G.C. Chamness and G.M. Clark, How to use prognostic factors in axillry nodenegative breast cancer patients [see comments], J. Natl. Cancer Inst. 82 (1990), 1006-1015.

[26] G.U. Auer, T.O. Caspersson and A. Wallgren, DNA content and survival in mammary carcinoma, Anal. Quant. Cytol. Histol. 2 (1980), 161-165.
[27] A. Chassevent, M.L. Jourdan, S. Romain, F. Descotes, M. Colonna, P.M. Martin, M. Bolla and F. Spyratos, S-phase fraction and DNAA ploidy in 633 T1T2 breast cancers: a standardized flow cytometric study, Clin. Cancer Res. 7 (2001), 909917.

[28] O.P. Kallioniemi, G. Blanco, M. Alavaikko, T. Hietanen, J. Mattila, K. Lauslathi and T. Koivula, Tumour DNA ploidy as an independent prognostic factor in breast cancer, $B r$. J. Cancer 56 (1987), 637-642.

[29] W.E. Lewis, Prognostic significance of flow cytometric DNA analysis in node-negative breast cancer patients, Cancer $\mathbf{6 5}$ (1990), 2315-2320.

[30] F. Canizares, J Sola, M. Perez, I. Tovar, M. De Las Heras, J. Salinas, R. Penafiel and P. Martinez, Preoperative values of CA 15-3 and CEA as prognostic factors in breast cancer: a multivariate analysis, Tumour Biol. 22 (2001), 273-281.

[31] M. Steinardottir, J.G. Jonasson, I. Petursdottir, H. Sigurdsson and H.M. Ogmundsdottir, A comparison of cytogenetic studies and flow cytometry in breast carcinomas, Cytometry 28 (1997), 323-328.

[32] D. Hansemann, Über die Anaplasie der Geschwulstzellen und die assymetrische Mitose, Virchows Arch. Pathol. Anat. 129 (1892), 436-449.

[33] M. Tubiana, M.H. Pejovic, S. Koscielny, N. Chavaudra and E. Malaise, Growth rate, kinetics of tumor cell proliferation and long-term outcome in human breast cancer, Int. J. Cancer $\mathbf{4 4}$ (1989), 17-22.

[34] O.P. Kallioniemi, Comparison of fresh and paraffin-embedded tissue as starting material for DNA flow cytometry and evaluation of intratumor heterogeneity, Cytometry 9 (1988), 164-169.

[35] H. Sigurdsson, B. Baldetrop, A. Borg, M. Dalberg, M. Fernö, D. Killander and H. Olsson, Indicators of prognosis in nodenegative breast cancer [see comments], N. Engl. J. Med. 322 (1990), 1045-1053.

[36] D.W. Visscher, R.J. Zarbo, G. Jakobsen, A. Kambouris, G. Talpos, W. Sakr and J.D. Crissman, Multiparametric deoxyribonucleic acid and cell cycle analysis of breast carcinomas by flow cytometry. Clinopathologic correlations, Lab. Invest. 62 (1990), 370-378.

[37] R.S. Camplejohn, C.M. Ash, C.E. Gillett, B. Raikundalia, D.M. Barnes, W.M. Gregory, M.A. Richards and R.R. Millis, The prognostic significance of DNA flow cytometry in breast cancer: results from 881 patients treated in a single centre, $B r . J$. Cancer 71 (1995), 140-145.

[38] S.M. O'Reilly, R.S. Camplejohn, D.M. Barnes, R.R. Millis, R.D. Rubens and M.A. Richards, Node-negative breast cancer: prognostic subgroups defined by tumor size and flow cytometry [see comments], J. Clin. Oncol. 8 (1990), 2040-2046. 


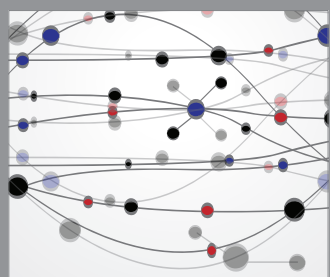

The Scientific World Journal
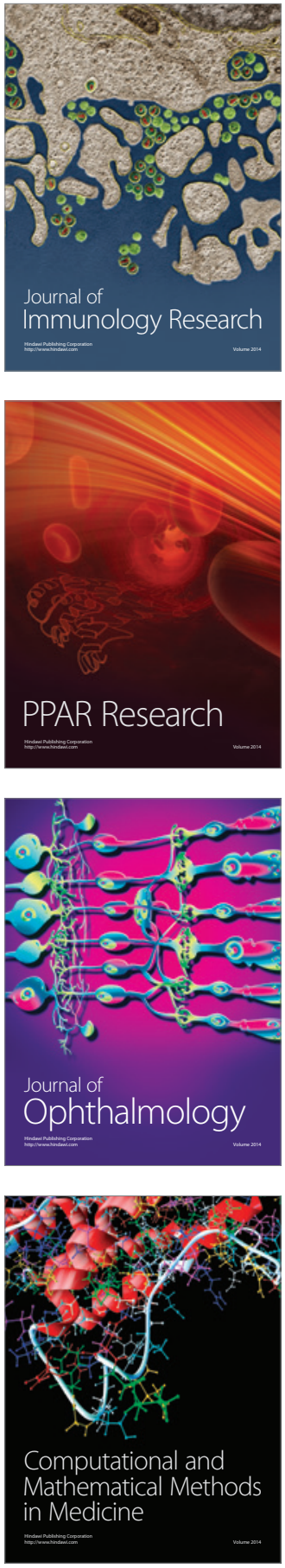

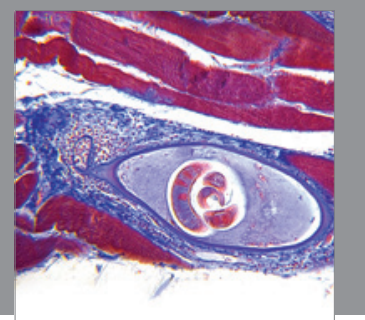

Gastroenterology

Research and Practice
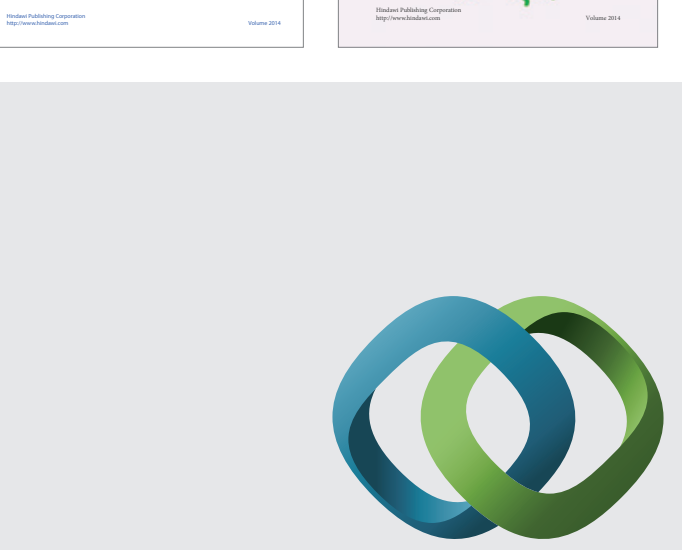

\section{Hindawi}

Submit your manuscripts at

http://www.hindawi.com
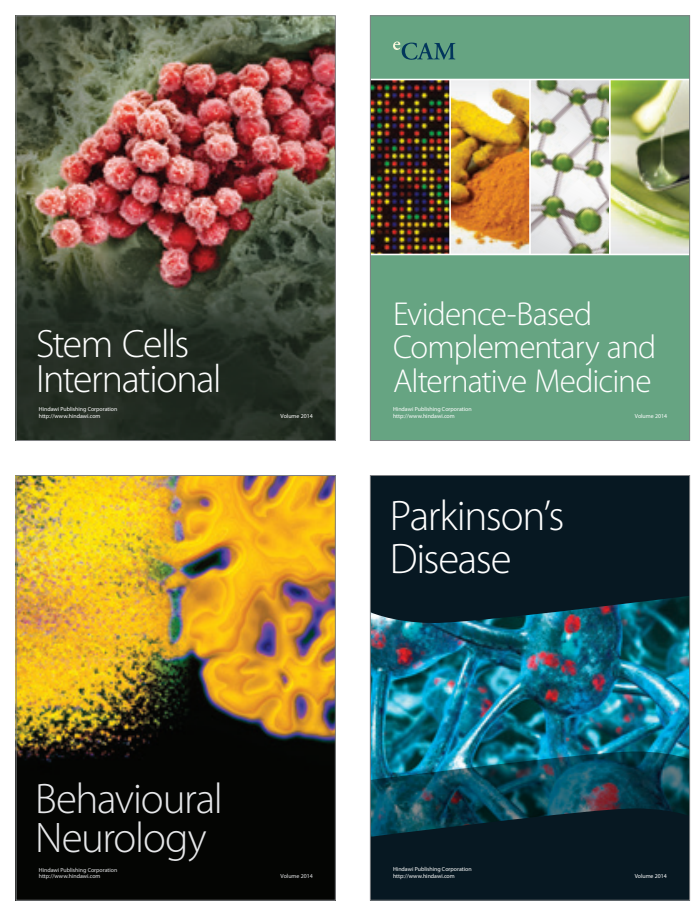

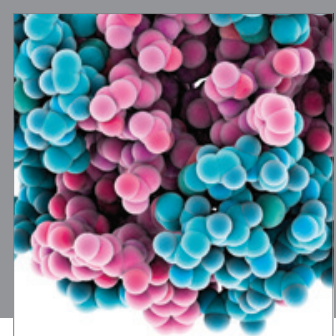

Journal of
Diabetes Research

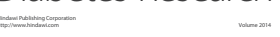

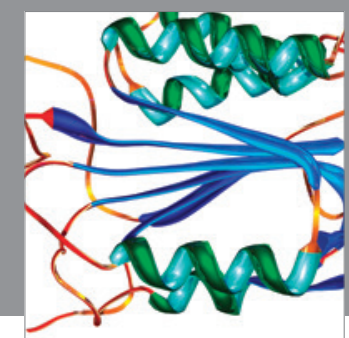

Disease Markers
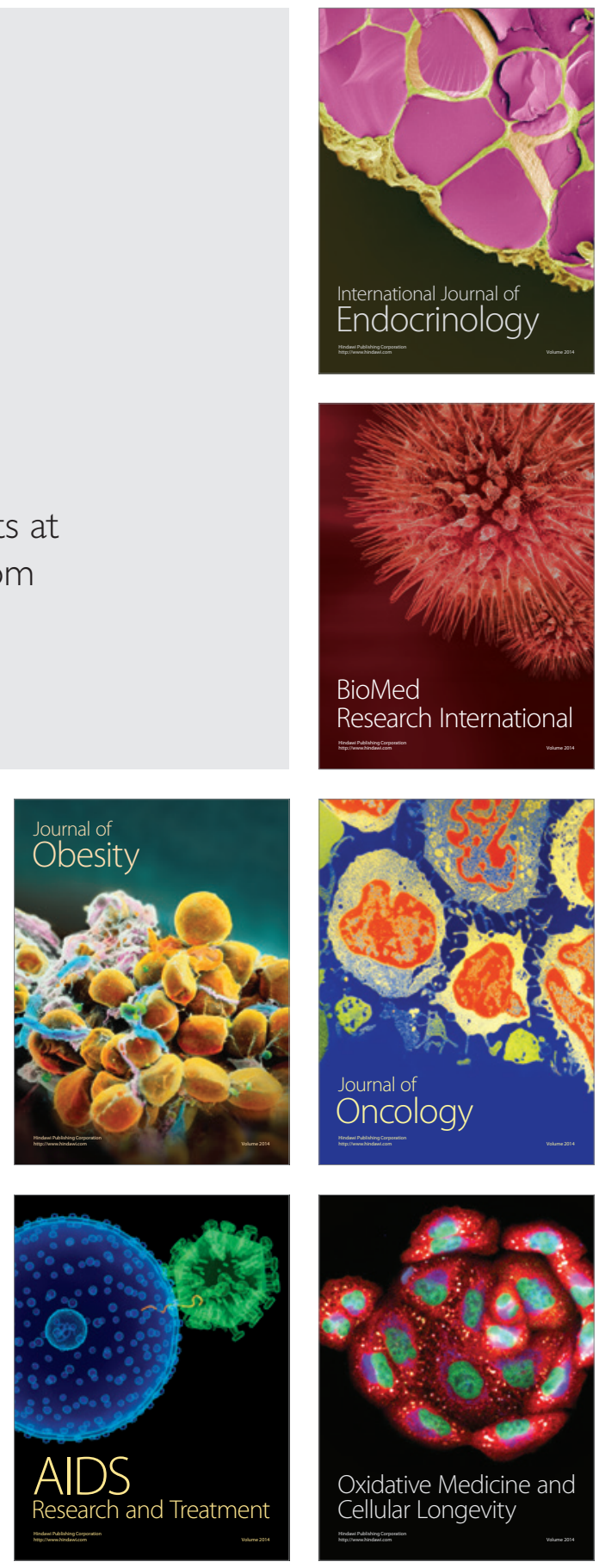